ITC 2/49

Information Technology and Control

Vol. 49 / No. 2 / 2020

pp. 302-316

DOI 10.5755/j01.itc.49.2.24612
Switching Stabilization for Nonlinear Networked Control Systems with Delays and Packet Losses

Received 2019/11/13

Accepted after revision 2020/03/22

HOW TO CITE: Zhang, Q., Liu, B. (2020). Switching Stabilization for Nonlinear Networked Control Systems with Delays and Packet Losses. Information Technology and Control, 49(1), 302-316. https://doi.org/10.5755/j01.itc.49.2.24612

\title{
Switching Stabilization for Nonlinear Networked Control Systems with Delays and Packet Losses
}

\section{Qi Zhang, Bin Liu}

Engineering Research Center for Metallurgical Automation and Measurement Technology of Ministry of Education; Wuhan, China

Hubei Province Key Laboratory of Systems Science in Metallurgical Process,Wuhan 430081, China; phone: 15671621305; email: 499319017@qq.com, liubin@wust.edu.cn

Corresponding author: 499319017@qq.com

This paper studies the stabilization problem for nonlinear networked control systems (NNCSs) with bilateral network-induced random delay and packet dropout. T-S fuzzy model is employed to represent the nonlinear controlled plant. Based on the T-S model, a discrete-time fuzzy switched system model with uncertain parameters is established by means of the uncertain method and switching system method. And an augmented matrix is used to represent the system model. Furthermore, the exponential stability condition for the state of the fuzzy switched system is obtained by using the combination of slow switching model-dependent average dwell time (MDADT) method and fast switching MDADT method. Finally, a series of rotary inverted pendulum (RIP) experiments are provided to illustrates the effectiveness of the proposed method and prove that the proposed fuzzy controller based on T-S fuzzy model can balance the rotary inverted pendulum in a greater state range rather than the linear controller based on linearization

KEYWORDS: nonlinear networked control systems, T-S fuzzy model, switched system, mode-dependent average dwell time. 


\section{Introduction}

With the rapid development of network communication technology and computer technology, network control systems (NCSs) came into being. Compared with the traditional control strategy, NCSs have several advantages, such as remote distributed control, remote distributed sharing, easy installation, easy maintenance and high flexibility [27]. However, the network-induced delays and packet dropouts would occur inevitably during the data transmission in NCSs, which would lower the control performance and even make NCSs unstable. A large number of methods such as input delay approach, Bernoulli distribution, Markov chain and switched system approach have been employed to model and analyze linear NCSs with random delays and random packet dropouts [27]. However, few paper have studied the nonlinear NCSs. It is necessary to study NNCSs subject to random delays and packet losses.

In general, nonlinear plants are usually linearized near the equilibrium point, and then the controller is analyzed and designed based on the linearized model. However, there is a large error between the linearized model and the original nonlinear model, which will degrade the control performance of the system. T-S fuzzy model is considered as a powerful technique to deal with nonlinear functions, and has been widely used to represent nonlinear system models [17]. In recent years, the analysis and synthesis of fuzzy-model-based nonlinear networked control systems have received increasing attentions [15]. The input delay approach has been effectively utilized to analyze the NCS. However, the input delay approach aims to obtain the maximum delay upper bound that the NCS can tolerate, and many results are based on more complex Lyapunov-Krasovskii function (LKF). Hu et al. [5] and Marouf et al. [14] represented the network-induced delays and packet dropouts by the input delay approach, and the stability of the NNCS based on T-S fuzzy model is studied. Zhi et al. [28] investigated the stabilization problem for T-S fuzzy system with interval time-varying delay by constructing a novel augmented LKF and proposed a developed reciprocally convex matrix inequality to bound the derivative of the LKF. In [20], a networked fuzzy control system was established based on event-triggered
T-S fuzzy model and the $\mathrm{H} \infty$ controller is obtained by parallel distributed compensation (PDC) technique. In [24], the probability of random delays and random packet dropouts in the NCS was taken as the fuzzy membership function, and a quasi-T-S fuzzy model is proposed to represent the NCS. In [13], a robust $\mathrm{H} \infty$ control problem for a networked switched fuzzy system was studied. The induced delay and packet dropout caused by network are dealt with LKFs and free-weighting matrices. Han et al. [2] and Lin et al. [9] described the event of packet dropouts in the NCS as Bernoulli distribution process, and utilize the stochastic system analysis approach to analyze the condition of mean square stability for the NNCS based on T-S fuzzy model. In [6], the problem of the fuzzy controller for NNCS with packet losses and parameter uncertainties was studied by using the interval type-2 fuzzy-model-based approach and a novel fuzzy controller to guarantee the closed-loop system to be stochastically stable with an optimal performance. $\mathrm{He}$ et al. [4] analyzed the Markovian jump systems with nonlinearity and time-varying delay and proposed an improved exponential stability criterion for the system.

Because the switched system approach can efficiently describe the different modes of the system due to the changes in the external environment, it is widely applied in power transmission systems, traffic control systems, flight control systems and other fields. In recent years, a number of scholars have studied and analyzed the NCS subject to delays and packet losses by mean of the switched system approach. In [7], the NCS with time-varying short delays was represented as an improve system according to the distribution of delays and design a mode-dependent state feedback controller wheres the packet dropout is not studied. In [26], the NCS with constant delays and random packet dropouts was modeled as a switched system, and exponential stability is investigated based on average dwell time (ADT). In [19], T-S fuzzy system with time-varying delays and random packet losses was modeled as a fuzzy switched system with input delays, and its exponential stability was analyzed by means of the $\mathrm{ADT}$ technique. Based on ADT, Zhang et al. [25] proposed slow switching model-depen- 
dent average dwell time (MDADT) approach and fast switching model-dependent average dwell time (MDADT) approach, and presented new stability results for a discrete-time switched system with unstable subsystems. Liu et al. [11] applied MDADT to T-S fuzzy model, but this paper only studied the openloop switched system. In [12], the NCS with bilateral random delays and packet losses was modeled as a discrete-time switched system model with parameter uncertainty, and the exponential stability of the system was considered by means of the slow-switching MDADT method. However, only the linear system was studied in the paper. Li and zhang [8] investigated robust $\mathrm{H} \infty$ stability of open-loop switched uncertain discrete-time fuzzy systems under slow-switching MDADT switching. Xue et al. [21] was concerned with the stabilization problem of type-2 fuzzy systems with network-induced packet losses. By regarding the packet lost process as an unstable mode of a switched system, the stability of the system was then guaranteed with the aid of the MDADT approach in the sense of the slow and fast switching. However, the paper did not consider the random delay existing in the system and assumed the control input was zero when the event of data dropout occurred.

Motivated by the above discussion, this paper describes the NNCS with bilateral random delays and random packet losses as a discrete-time fuzzy switched system model with uncertain parameters. Based on multiple Lyapunov functions (MLFs) and model-dependent average dwell time (MDADT) in the sense of the slow and fast switching, the exponential stability of the network switched system are analyzed. And the fuzzy state feedback controller gains are derived by solving linear matrix inequalities (LMIs). Finally, a series of rotary inverted pendulum (RIP) experiments are provided to illustrate the feasibility of the proposed method and verify that the fuzzy controller based on T-S fuzzy model can balance the RIP in a larger stability range than the linear controller based on model linearization.

The contributions of this paper can be summarized as follows: (1) a new discrete-time fuzzy switched system model with uncertain parameters is proposed to described the NNCS with random delay and packet losses; (2) the exponential stability condition for the system is analyzed by utilizing MDADT approach in the sense of the slow and fast switching; (3) the fuzzy controller gains of the closed system are derived by solving LMIs.

\section{Problem Formulation and System Model}

In this section, T-S fuzzy model is introduced to represent the nonlinear plant in the NCS subject to random delays and random packet losses. The nonlinear controlled plant in the NCS is considered as

$$
\dot{x}(t)=f(x(t))+g(x(t), \quad v(t)),
$$

where $x(t) \in \mathfrak{R}^{n}$ is the state vector and $v(t)$ is the control input. $f(x(t))$ and $g(x(t), v(t))$ are known nonlinear functions. The nonlinear system in Equation (1) can be represented by a T-S fuzzy model as follows in the IF-THEN form

Rule $i$ : IF $\theta_{1}(t)$ is $M_{i 1}, \ldots, \theta_{g}(t)$ is $M_{i g}$

\section{THEN}

$\dot{x}(t)=A_{i} x(t)+B_{i} v(t), i=1,2, \cdots, r$,

where $\theta_{1}(t), \theta_{2}(t), \cdots, \theta_{g}(t)$ are the premise variables, $g$ is the number of premise variables; $M_{i j}(i=1, \cdots r, j=1, \cdots g)$ is the fuzzy set; $r$ is the number of rules; $A_{i}$ and $B_{i}$ are the known system matrices with appropriate dimensions.

Therefore, the nonlinear system (1) can be described as

$$
\dot{x}(t)=\sum_{i=1}^{r} \mu_{i}(\theta(t))\left(A_{i} x(t)+B_{i} v(t)\right),
$$

where

$$
\begin{aligned}
& \mu_{i}(\theta(t))=\frac{\varpi_{i}(\theta(t))}{\sum_{i=1}^{r} \varpi_{i}(\theta(t))}, \varpi_{i}(\theta(t))=\prod_{j=1}^{g} M_{i j}\left(\theta_{j}(t)\right), \\
& \theta(t)=\left[\begin{array}{llll}
\theta_{1}(t), & \theta_{2}(t), & \cdots, & \theta_{g}(t)
\end{array}\right]
\end{aligned}
$$

and $M_{i j}\left(\theta_{j}(t)\right)$ is the degree of membership of $\theta_{j}(t)$ in $M_{i j}$. Usually, $0 \leq \varpi_{i}(\theta(t)) \leq 1$ for $i=1,2, \cdots, r$ and $\sum_{i=1}^{r} \varpi_{i}(\theta(t))>0$. Therefore, $\mu_{i}(\theta(t)) \geq 0$ and $\sum_{i=1}^{r} \mu_{i}(\theta(t))=1$.

The NNCS subject to bilateral random delays and random packet losses is shown in Figure 1. 


\section{Figure 1}

The structure diagram of the NNCS

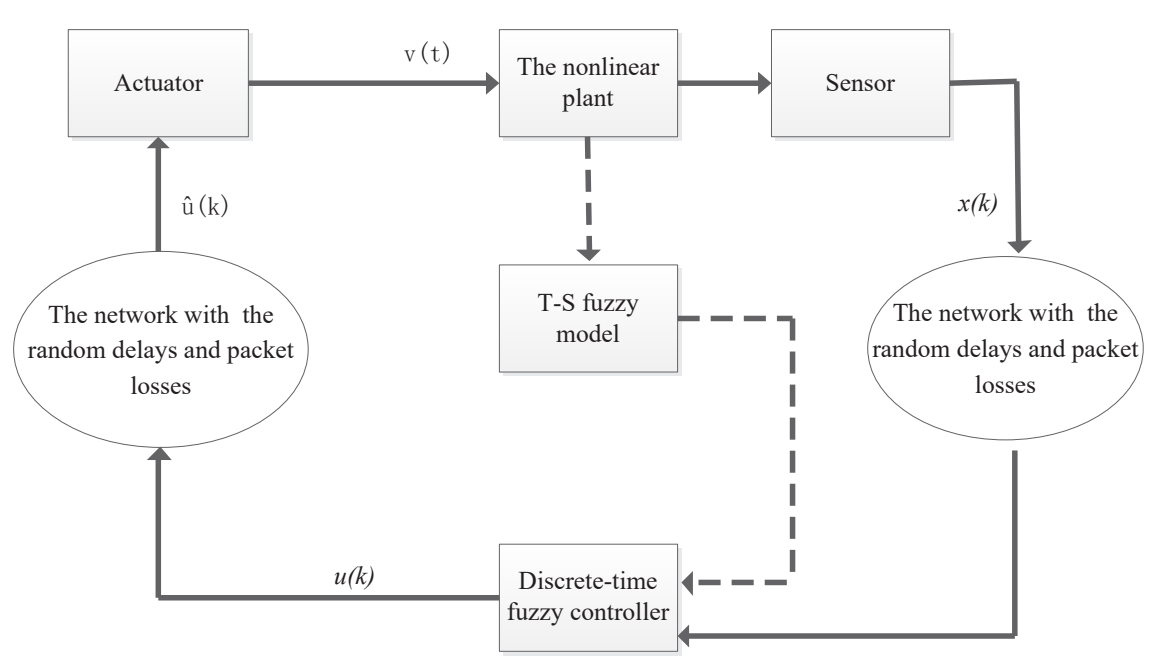

The assumptions are given as follows:

1 The sensor is time-driven and its sampling period is $\mathrm{T}$.

2 Both the controller and the actuator are event-driven. The actual input of the system is realized via a zero-order hold device. When the event of packet loss occurs in the system (between the sensor and the controller or between the controller and the actuator), the actuator data will not be updated during the current sampling period.

3 The random network-induced delay satisfies $\tau_{k}=\tau_{k}^{s c}+\tau_{k}^{c a} \in(0, T)$, and $\tau_{k}^{s c}$ is sensor-to-controller delay while $\tau_{k}^{c a}$ is the controller-to-actuator delay.

The signal timing of the NCS is shown in figure 2. By considering the network-induced delays, the control input is [18]

$$
v(t)=\left\{\begin{array}{l}
\hat{u}(k-1), \quad k T<t \leq k T+\tau_{k}, \\
\hat{u}(k), \quad k T+\tau_{k}<t \leq(k+1) T,
\end{array}\right.
$$

where

$\hat{u}(k)=\left\{\begin{array}{l}\hat{u}(k-1), \text { packet }- \text { dropout }, \\ u(k), \quad \text { packet }- \text { send } .\end{array}\right.$

When the event of packet loss does not occurs within the current sampling interval, such as $[k T,(k+1) T]$,
Figure 2

The signal timing diagram of the NCS

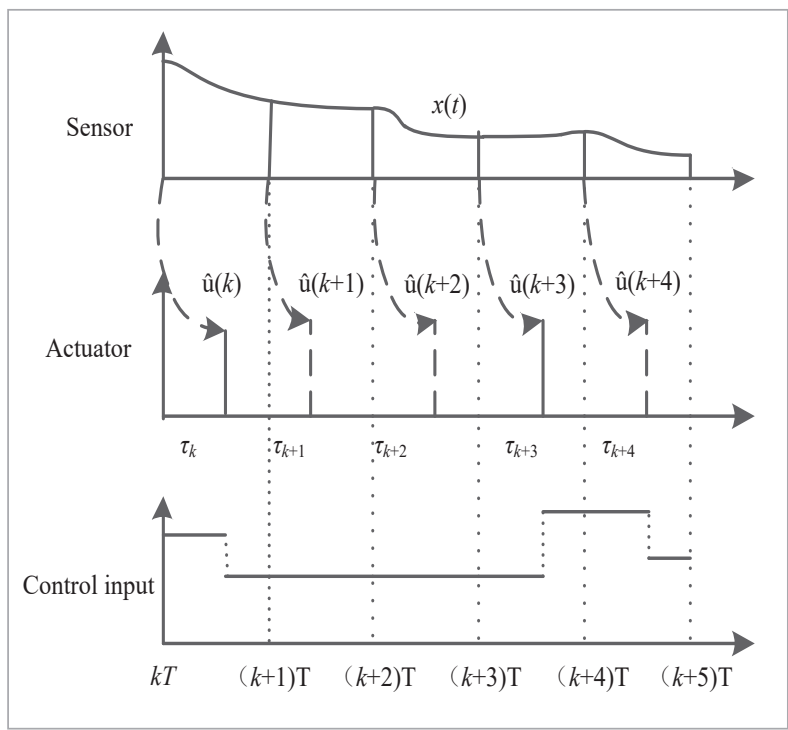

$[(k+3) T,(k+4) T]$ and $[(k+4) T,(k+5) T]$. The random delays exist, so the corresponding discrete-time model of the fuzzy system (3) is

$$
S_{0}: x(k+1)=\sum_{i=1}^{r} \mu_{i}(\theta(k))\left(\begin{array}{r}
A_{d i} x(k)+B_{d 0 i}\left(\tau_{k}\right) u(k) \\
+B_{d 1 i}\left(\tau_{k}\right) \hat{u}(k-1)
\end{array}\right),
$$


where $A_{d i}=e^{A_{i} T}, B_{d 0 i}\left(\tau_{k}\right)=\int_{0}^{T-\tau_{k}} e^{A_{i} s} d s \cdot B_{i}, \quad B_{d 1 i}\left(\tau_{k}\right)=$ $\int_{T-\tau_{k}}^{T} e^{A_{i} s} d s \cdot B_{i}$.

Within the sampling interval $[(k+1) T,(k+2) T]$ and $[(k+2) T,(k+3) T]$, the event of packet loss occurs. The corresponding discrete-time model of the fuzzy system (3) is

$$
S_{1}: x(k+1)=\sum_{i=1}^{r} \mu_{i}(\theta(k))\left(A_{d i} x(k)+B_{d 2 i} \hat{u}(k-1)\right),
$$

where $B_{d 2 i}=\int_{0}^{T} e^{A_{i} s} d s \cdot B_{i}$.

Let $z(k)=\left[\begin{array}{ll}x^{T}(k) & \hat{u}^{T}(k-1)\end{array}\right]^{T}$ denote the system augmented matrix and $\sigma(k) \in \Omega=\{0,1\}$ denote system switching signal. Therefore, the system models (5) and (6) can be described as the following discrete-time fuzzy switched system model

$$
S_{\sigma(k)}: z(k+1)=\sum_{i=1}^{r} \mu_{i}(\theta(k))\left(\Phi_{\sigma(k) i} x(k)+\Gamma_{\sigma(k) i} u(k)\right) \cdot(7)
$$

When $\sigma(k)=0$, the event of the packet loss does not occur in the current sampling, there is

$\Phi_{0 i}=\left[\begin{array}{cc}A_{d i} & B_{d 1 i}\left(\tau_{k}\right) \\ 0 & 0\end{array}\right], \Gamma_{0 i}=\left[\begin{array}{c}B_{d 0 i}\left(\tau_{k}\right) \\ I\end{array}\right]$.

When $\sigma(k)=1$, the event of the packet loss occurs in the current sampling, there is

$\Phi_{1 i}=\left[\begin{array}{cc}A_{d i} & B_{d 2 i} \\ 0 & I\end{array}\right], \Gamma_{1 i}=0$.

For the random delays in the system, the uncertainty method is used to deal with it. Define

$$
\begin{aligned}
& \bar{F}_{i}\left(\tau_{k}^{\prime}\right)=\int_{0}^{-\tau_{k}^{\prime}} e^{A_{i} s} d s, \tau_{k}^{\prime}=\tau_{k}-T / 2 \in(-T / 2, T / 2), \\
& \sigma_{i}>\max _{\tau_{k}^{\prime} \in(-T / 2, T / 2)}\left\|\bar{F}_{i}\left(\tau_{k}^{\prime}\right)\right\|_{2}=\left\|\int_{0}^{-\tau_{k}^{\prime}} e^{A_{i} s} d s\right\|_{2}, \\
& B_{d 0 i}=\int_{0}^{T / 2} e^{A_{i} s} d s \cdot B_{i}, B_{d 1 i}=\int_{T / 2}^{T} e^{A_{i} s} d s \cdot B_{i}, D_{i}=\sigma_{i} e^{A_{i} T}, \\
& E_{i}=B_{i}, F_{i}\left(\tau_{k}^{\prime}\right)=\sigma_{i}^{-1} \int_{0}^{-\tau_{k}^{\prime}} e^{A_{i} s} d s .
\end{aligned}
$$

Then there is

$$
\begin{aligned}
& B_{d 0 i}\left(\tau_{k}\right)=\int_{0}^{T-\tau_{k}} e^{A_{i} s} d s \cdot B_{i}=B_{d 0 i}+D_{i} F_{i}\left(\tau_{k}^{\prime}\right) E_{i}, \\
& B_{d 0 i}\left(\tau_{k}\right)=\int_{T-\tau_{k}}^{T} e^{A_{i} s} d s \cdot B_{i}=B_{d 1 i}-D_{i} F_{i}\left(\tau_{k}^{\prime}\right) E_{i}, \\
& F_{i}^{T}\left(\tau_{k}^{\prime}\right) F_{i}\left(\tau_{k}^{\prime}\right)=\sigma_{i}^{-2} \bar{F}_{i}^{T}\left(\tau_{k}^{\prime}\right) \bar{F}_{i}\left(\tau_{k}^{\prime}\right)<I .
\end{aligned}
$$

According to the parallel distribution compensation (PDC) [17], the fuzzy state feedback controller is designed by the state information of augmented matrix $z(k)$ as follows

$$
u(k)=\sum_{i=1}^{r} \mu_{i}(\theta(k)) K_{i} z(k)
$$

where $K_{i}$ is the controller gain matrices determined later.

By substituting Equation (8) into Equation (7), the closed-loop system model with uncertain parameters can be obtained as follows

$$
S_{\sigma(k)}: z(k+1)=\sum_{i=1}^{r} \sum_{j=1}^{r} \mu_{i}(\theta(k)) \mu_{j}(\theta(k))\left(\hat{\Phi}_{\sigma(k) i j} z(k)\right),
$$

where $\hat{\Phi}_{0 i j}=\tilde{\Phi}_{0 i}+\tilde{\Gamma}_{0 i} K_{j}+\tilde{D}_{i} F_{i}\left(\tau_{k}^{\prime}\right)\left(\tilde{E}_{i}+E_{i} K_{j}\right)$, $\hat{\Phi}_{1 i j}=\Phi_{1 i}, \tilde{\Phi}_{0 i}=\left[\begin{array}{cc}A_{d i} & B_{d 1 i} \\ 0 & 0\end{array}\right], \tilde{\Gamma}_{0 i}=\left[\begin{array}{c}B_{d 0 i} \\ I\end{array}\right], \tilde{D}_{i}=\left[\begin{array}{c}D_{i} \\ 0\end{array}\right]$, $\tilde{E}_{i}=\left[\begin{array}{ll}0 & -E_{i}\end{array}\right]$.

For $\sigma(k) \in \Omega=\{0,1\}, S_{0}$ denotes the subsystem mode where the packet is transmitted successfully during the current sampling period and $S_{1}$ denotes the subsystem mode where the event of packet-dropout occurs during the current sampling period. According to the switched system (9), the subsystem $S_{0}$ can be stabilized by means of a proper controller when the open-loop system is unstable. However, the subsystem $S_{1}$ is uncontrolled, and the subsystem $S_{1}$ cannot be stabilized when the open-loop system is unstable. Therefore, according to whether the event of packet-dropout occurs, the system can be described as a whole switched system model with a stable subsystem $S_{0}$ and an unstable subsystem $S_{1}$. The fuzzy switched system model with uncertain parameters presented in this paper can effectively describe the NNCS with random delays and random packet losses.

\section{The System Exponential Stability Analysis}

Definition 1. The equilibrium $x=0$ of system (9) is global uniformly exponential stable (GUES) under a certain switching signal $\sigma(k)$ if for any initial condition $x\left(k_{0}\right)$, there exist constant $a>0$ and $0<\gamma<1$ such that $\|x(k)\| \leq a \gamma^{\left(k-k_{0}\right)}\left\|x\left(k_{0}\right)\right\|, \forall k \geq k_{0}$ and the exponential decay rate is $\gamma$.

Definition 2. (Slow switching MDADT) [25]. For any $k_{2}>k_{1} \geq 0, p \in \Omega=\{0,1\}$, over the interval $\left[k_{1}, k_{2}\right]$, 
let $N_{\sigma p}\left(k_{1}, k_{2}\right)$ denote the numbers of the subsystem $S_{p}$ activated, $T_{p}\left(k_{1}, k_{2}\right)$ denote the overall running time period of the subsystem $S_{p}$, and $N_{0 p}$ denote the mode-dependent chatter bounds. If there exists $\tau_{a p}>0$ such that the following holds

$N_{\sigma p}\left(k_{1}, k_{2}\right) \leq N_{0 p}+T_{p}\left(k_{1}, k_{2}\right) / \tau_{a p}$,

then $\tau_{a p}$ is MDADT of the subsystem $S_{p}$.

Definition 3. (Fast switching MDADT) [25]. For any $k_{2}>k_{1} \geq 0, p \in \Omega=\{0,1\}$, over the interval $\left[k_{1}, k_{2}\right]$, let $N_{\sigma p}\left(k_{1}, k_{2}\right)$ denote the numbers of the fast switching subsystem $S_{p}$ activated, $T_{p}\left(k_{1}, k_{2}\right)$ denote the overall running time period of the fast switching subsystem $S_{p}$, and $N_{0 p}$ denote the mode-dependent chatter bounds. If there exists $\tau_{a p}>0$ such that the following holds

$N_{\sigma p}\left(k_{1}, k_{2}\right) \geq N_{0 p}+T_{p}\left(k_{1}, k_{2}\right) / \tau_{a p}$

then $\tau_{a p}$ is called fast switching MDADT of the subsystem $S_{p}$.

Remark 1. The idea of slow switching MDADT is aimed at that for the switched system, the time interval between two consecutive switching points of a subsystem $S_{p}$ may be less than $\tau_{a p}$ sampling period, but the average time interval between two consecutive switching points of a subsystem $S_{p}$ is not less than $\tau_{a p}$ sampling period. Similarly, the idea of fast switching MDADT is aimed at that for the switched system (9), the time interval between two consecutive switching points of a subsystem $S_{p}$ may be larger than $\tau_{a p}$ sampling period, but the average time interval between two consecutive switching points of a subsystem $S_{p}$ is not larger than $\tau_{a p}$ sampling period.

From the above definition, in [12], all subsystems follow the slow switching scheme. However, in this paper, the stable subsystem $S_{0}$ follows the slow switching scheme while the unstable subsystem $S_{1}$ follows the fast switching scheme. The main idea is to design switching scheme so that the MDADT of the stable subsystem $S_{0}$ is large enough and the MDADT of the unstable subsystem $S_{1}$ is small enough, and then guarantee the whole switched system (9) GUES. Therefore, over the interval $\left[k_{1}, k_{2}\right]$, for the stable subsystem $S_{0}$ and the unstable subsystem $S_{1}$, there are $N_{\sigma 0}\left(k_{1}, k_{2}\right) \leq N_{00}+T_{0}\left(k_{1}, k_{2}\right) / \tau_{a 0}$ and $N_{\sigma 1}\left(k_{1}, k_{2}\right) \geq N_{01}+T_{1}\left(k_{1}, k_{2}\right) / \tau_{a 1}$.

Lemma 1. Consider the discrete-time fuzzy switched system (9), let $-1<\lambda_{0}<0, \quad \lambda_{1}>0$ be given constants.
Suppose there exist $\mathrm{C}^{1}$ functions $V_{0}(z(k)): R^{n} \rightarrow R$, $V_{1}(z(k)): R^{n} \rightarrow R$ and class $\kappa_{\infty}$ function $\kappa_{1} 、 \kappa_{2}$, such that

$$
\begin{aligned}
& \left\{\begin{array}{l}
\kappa_{1}(\|z(k)\|) \leq V_{0}(z(k)) \leq \kappa_{2}(\|z(k)\|) \\
\kappa_{1}(\|z(k)\|) \leq V_{1}(z(k)) \leq \kappa_{2}(\|z(k)\|)
\end{array}\right. \\
& \left\{\begin{array}{l}
\Delta V_{0}(z(k)) \leq \lambda_{0} V_{0}(z(k)) \\
\Delta V_{1}(z(k)) \leq \lambda_{1} V_{1}(z(k))
\end{array}\right.
\end{aligned}
$$

and $\forall\left(\sigma\left(k_{i}\right)=p, \sigma\left(k_{i}^{-}\right)=q\right) \in \Omega \times \Omega, p \neq q$, there exist constants $\mu_{0}>1$ and $0<\mu_{1}<1$, such that

$$
\left\{\begin{array}{l}
V_{0}\left(z\left(k_{i}\right)\right) \leq \mu_{0} V_{1}\left(z\left(k_{i}^{-}\right)\right) \\
V_{1}\left(z\left(k_{i}\right)\right) \leq \mu_{1} V_{0}\left(z\left(k_{i}^{-}\right)\right)
\end{array},\right.
$$

then the system (9) is GUES with marginal $\gamma$ under any switching signal with the following MDADT:

$$
\left\{\begin{array}{l}
\tau_{a 0} \geq \tau_{a 0}^{*}=-\frac{\ln \mu_{0}}{\ln \left(1+\lambda_{0}\right)} \quad\left(-1<\lambda_{0}<0\right) \\
\tau_{a 1} \leq \tau_{a 1}^{*}=-\frac{\ln \mu_{1}}{\ln \left(1+\lambda_{1}\right)} \quad\left(\lambda_{1}>0\right)
\end{array},\right.
$$

where $\tau_{a 0}$ is MDADT of the subsystem $S_{0}$ while $\tau_{a 1}$ is MDADT of the subsystem $S_{1}$.

The exponential stability conditions for the networked switched system (9) are presented in the following theorem.

Theorem 1. Consider the discrete-time fuzzy switched system (9). Let $\mu_{0}>1,0<\mu_{1}<1,-1<\lambda_{0}<0$, $0<\lambda_{1}<1$ be given constants. If there exist matrices $P_{p}>0, P_{q}>0, \forall(p, q) \in \Omega \times \Omega, p \neq q$, such that

$$
V_{p}(z(k+1)) \leq\left(1+\lambda_{p}\right) V_{p}(z(k)),
$$

$$
P_{p}<\mu_{p} P_{q},
$$

then the system (9) is GUES with decay rate $\gamma=\max \left\{\left(\mu_{0}^{\frac{1}{\tau_{0} 0}} \bar{\lambda}_{0}\right),\left(\mu_{1}^{\frac{1}{\tau_{a 1}}} \bar{\lambda}_{1}\right)\right\}$ when the MDADT of the switching signal satisfies (10).

Proof: Denote $V_{\sigma\left(k_{i}\right)}\left(z\left(k_{i}\right)\right)=V_{\sigma\left(k_{i}\right)}\left(k_{i}\right), \bar{\lambda}_{p}=1+\lambda_{p}$, $p \in \Omega$. Consider the Lyapunov function as follows

$V_{p}(k)=\left\{\begin{array}{ll}z^{T}(k) P_{0} z(k), & p=0 \\ z^{T}(k) P_{1} z(k), & p=1\end{array}\right.$. 
Let $k_{0}, k_{1}, k_{2}, \cdots, k_{i}, \cdots, k_{i+1}, \cdots, k_{N_{\sigma}(n T, 0)}$ denote the switching times on time interval $[0, n T]$. From Equations (11) and (12), there is

$$
\begin{aligned}
V_{\sigma\left(k_{i}\right)}(k) & <\bar{\lambda}_{\sigma\left(k_{i}\right)}^{\left(k-k_{i}\right)} V_{\sigma\left(k_{i}\right)}\left(k_{i}\right) \\
& <\mu_{\sigma\left(k_{i}\right)} \bar{\lambda}_{\sigma\left(k_{i}\right)}^{\left(k-k_{i}\right)} V_{\sigma\left(k_{i}^{-}\right)}\left(k_{i}^{-}\right) \\
& <\mu_{\sigma\left(k_{i}\right)} \bar{\lambda}_{\sigma\left(k_{i}\right)}^{\left(k-k_{i}\right)} \bar{\lambda}_{\sigma\left(k_{i-1}\right)}^{\left(k_{i-1}\right)} V_{\sigma\left(k_{i-1}\right)}\left(k_{i-1}\right) \\
& <\mu_{\sigma\left(k_{i}\right)} \mu_{\sigma\left(k_{i-1}\right)} \bar{\lambda}_{\sigma\left(k_{i}\right)}^{\left(k-k_{i}\right)} \bar{\lambda}_{\sigma\left(k_{i-1}\right)}^{\left(k_{i}-k_{i-1}\right)} V_{\sigma\left(k_{i-1}\right)}\left(k_{i-1}^{-}\right) \\
& \vdots \\
& <\prod_{s=1}^{i} \mu_{\sigma\left(k_{s}\right)} \bar{\lambda}_{\sigma\left(k_{i}\right)}^{\left(k-k_{i}\right)} \bar{\lambda}_{\sigma\left(k_{i-1}\right)}^{\left(k_{i}-k_{i-1}\right)} \ldots \bar{\lambda}_{\sigma\left(k_{i}\right)}^{\left(k_{2}-k_{i}\right)} \times \bar{\lambda}_{\sigma\left(k_{0}\right)}^{\left(k_{i}-k_{0}\right)} V_{\sigma\left(k_{0}\right)}\left(k_{0}\right)
\end{aligned}
$$

Let $k_{0}=0, V_{\sigma(k)}(k)=V_{\sigma\left(k_{i}\right)}(k)$, we have

$$
\begin{aligned}
& V_{\sigma(k)}(k) \\
& \leq \mu_{0}^{N_{\sigma 0(k, 0)}} \mu_{1}^{N_{\sigma(k, k)}} \times \bar{\lambda}_{0}^{T_{0}(k, 0)} \bar{\lambda}_{1}^{T_{1}(k, 0)} V_{\sigma(0)}(0)
\end{aligned}
$$

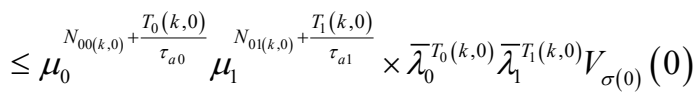

$$
\begin{aligned}
& =\exp \left\{N_{00(k, 0)} \ln \mu_{0}+N_{01(k, 0)} \ln \mu_{1}\right\}\left(\begin{array}{l}
\left(\mu_{0}^{\frac{1}{\tau_{\alpha 0}}} \bar{\lambda}_{0}\right)^{T_{0}(k, 0)} \times \\
\left(\frac{1}{\mu_{1}^{\tau_{\alpha 1}}} \bar{\lambda}_{1}\right)^{T_{1}(k, 0)} V_{\sigma(0)}(0)
\end{array}\right) \\
& \leq \exp \left\{N_{00(k, 0)} \ln \mu_{0}+N_{01(k, 0)} \ln \mu_{1}\right\} \gamma^{k} V_{\sigma(0)}(0)
\end{aligned}
$$

where $T_{0}(k, 0)$ is total running time of the subsystem $S_{0}$ and $T_{1}(k, 0)$ is total running time of the subsystem $S_{1}$. From $0<\mu_{0}^{\overline{\tau_{a 0}}} \bar{\lambda}_{0}<1 、 0<\mu_{1}^{\frac{1}{\tau_{a 1}}} \bar{\lambda}_{1}<1$, the MDADT of the switching signal satisfy (10). Set $L=\exp \left\{N_{0 p(k, 0)} \ln \mu_{p}+N_{01(k, 0)} \ln \mu_{1}\right\}$, $\gamma=\max \left\{\left(\mu_{0}^{\frac{1}{\tau_{\alpha 0}}} \overline{\lambda_{0}}\right),\left(\mu_{1}^{\frac{1}{\tau_{\alpha 1}}} \overline{\lambda_{1}}\right)\right\}$. Therefore, we have $V_{\sigma(k)}(k)<L \gamma^{k} V_{\sigma(0)}(0)$.

Denote $\beta_{1}=\min _{\forall p \in \Omega} \lambda_{\min }\left(P_{p}\right), \beta_{2}=\max _{\forall p \in \Omega} \lambda_{\max }\left(P_{p}\right)$, there is $\beta_{1}\|z(k)\| \leq V_{\sigma(k)}(k)<L \gamma^{k} V_{\sigma(0)}(0) \leq L \gamma^{k} \cdot \beta_{2}\|z(0)\|$.

Denote $a=\frac{L \beta_{2}}{\beta_{1}}$ and suppose that there is $u(-1)=0$ in the initial moment, we have

$$
\|x(k)\| \leq\|z(k)\|<a \gamma^{k}\|z(0)\|=a \gamma^{k}\|x(0)\| .
$$

When $\gamma<1$, the state of the system is GUES.

\section{The Controller Design for the System to Be Exponential Stable}

Lemma 2. (Schur complement formula) For given the constant matrices $\mathrm{A}, \mathrm{B}$ and $\mathrm{C}$, where $\mathrm{A}$ and $\mathrm{C}$ is symmetric matrix, $A+B^{T} C B<0$ is equivalent to $\left[\begin{array}{cc}A & B^{T} \\ B & -C^{-1}\end{array}\right]<0$ or $\left[\begin{array}{cc}-C^{-1} & B \\ B^{T} & A\end{array}\right]<0$

Lemma 3. For given the constant matrices $W, D, E$ and $F(k)$, where $W$ is symmetric matrix, for any $F(k)$ which satisfies $F^{T}(k) F(k)<I$, there would be $W+D F(k) E+E^{T} F(k)^{T} D^{T}<0$ only when there exist $\varepsilon>0$ and $W+\varepsilon D D^{T}+\varepsilon^{-1} E^{T} E<0$.

Lemma 4. For given the constant symmetric matrices $X_{i} 、 Y_{i}(1 \leq i \leq r)$ and the matrix $S>0$ with appropriate dimensions, there is following inequality

$2 \sum_{i=1}^{r} \sum_{j=1}^{r} \sum_{l=1}^{r} \sum_{m=1}^{r} h_{i} h_{j} h_{l} h_{m} X_{i j}^{T} S Y_{l m} \leq \sum_{i=1}^{r} \sum_{j=1}^{r} h_{i} h_{j}\left(X_{i j}^{T} S X_{i j}+Y_{i j}^{T} S Y_{i j}\right)$ An existence condition for the controllers is presented in the following theorem based on the stability condition in Theorem 1.

Theorem 2. Consider the discrete-time fuzzy switched system (9), given constants $\mu_{0}>1,0<\mu_{1}<1$, $-1<\lambda_{0}<0,0<\lambda_{1}<1$, if there exist the symmetric positive definite matrix $X_{p}, \quad p \in \Omega$, the matrix $Y_{0 i}$ and the scalars $\varepsilon_{i}>0$ for $i=1, \cdots, r$, such that

$\left\{\begin{array}{l}\Psi_{i i}<0, \\ \Psi_{i j}+\Psi_{j i}<0, \quad i=1, \cdots, r\end{array}\right.$

$$
\left[\begin{array}{cc}
-\left(1+\lambda_{p}\right) X_{p} & * \\
\hat{\Phi}_{p i} X_{p} & -X_{p}
\end{array}\right]<0, \quad i=1, \cdots, r \quad p=1, \text { (15) }
$$

$\left[\begin{array}{cc}-\mu_{p} X_{q} & * \\ X_{q} & -X_{p}\end{array}\right]<0, \quad \forall p \neq q \in \Omega$.

where

$$
\Psi_{i j}=\left[\begin{array}{ccc}
-\left(1+\lambda_{0}\right) X_{0} & * & * \\
\tilde{\Phi}_{0 i} X_{0}+\tilde{\Gamma}_{0 i} Y_{0 j} & \varepsilon_{i} \tilde{D}_{i} \tilde{D}_{i}^{T}-X_{0} & * \\
\tilde{E}_{i} X_{0}+E_{i} Y_{0 j} & 0 & -\varepsilon_{i} I
\end{array}\right] .
$$

Then the system (9) is GUES with decay rate $\gamma=\max \left\{\left(\mu_{0}^{\frac{1}{\tau_{a 0}}} \overline{\lambda_{0}}\right),\left(\mu_{1}^{\frac{1}{\tau_{1} 1}} \overline{\lambda_{1}}\right)\right\}$ when the MDADT of 
the switching signal satisfy (10) and the controller gain parameters satisfy $K_{j}=Y_{0 j} X_{0}^{-1}$.

Proof:

Denote $\sum_{i=1}^{r} \mu_{i}(\theta(k))=\sum_{i=1}^{r} \mu_{i}$, substituting Equation (9) into Equation (11), from Lemma 4, we can have

$\sum_{i=1}^{r} \sum_{j=1}^{r} \mu_{i} \mu_{j} \hat{\Phi}_{\sigma(k) i j}^{T} P_{p} \hat{\Phi}_{\sigma(k) i j}-\left(1+\lambda_{p}\right) P_{p}<0$

Applying Lemma 2 to Equations (12) and (17), there is

$$
\sum_{i=1}^{r} \sum_{j=1}^{r} \mu_{i} \mu_{j}\left[\begin{array}{cc}
-\left(1+\lambda_{p}\right) P_{p} & * \\
\hat{\Phi}_{\sigma(k) i j} & -P_{p}^{-1}
\end{array}\right]<0, \quad \forall p \in \Omega,
$$

$$
\left[\begin{array}{cc}
-\mu_{p} P_{q} & * \\
I & -P_{p}^{-1}
\end{array}\right]<0, \quad \forall p \neq q \in \Omega .
$$

Defining $X_{p}=P_{p}^{-1}, X_{q}=P_{q}^{-1}$, for $p, q \in \Omega$, preand post-multiplying Equation (18) by $\operatorname{diag}\left(X_{p}, I\right)$ and pre- and post-multiplying Equation (19) by $\operatorname{diag}\left(X_{q}, I\right)$, the Equations (18) and (19) are equivalent to

$$
\frac{\sum_{i=1}^{r} \sum_{j=1}^{r} \mu_{i} \mu_{j}\left[\begin{array}{cc}
-\left(1+\lambda_{p}\right) X_{p} & * \\
\hat{\Phi}_{p i j} X_{p} & -X_{p}
\end{array}\right]<0, \quad \forall p \in \Omega,}{\left[\begin{array}{cc}
-\mu_{p} X_{q} & * \\
X_{q} & -X_{p}
\end{array}\right]<0, \quad \forall p \neq q \in \Omega}
$$

Therefore, the Equation (16) is derived from Equation (21).

When $p=1$, the event of packet dropout occurs. From the system (9), there is $\hat{\Phi}_{p i j}=\Phi_{p i}$, the Equation (20) can be simplified to

$$
\sum_{i=1}^{r} \mu_{i}\left[\begin{array}{cc}
-\left(1+\lambda_{p}\right) X_{p} & * \\
\hat{\Phi}_{p i} X_{p} & -X_{p}
\end{array}\right]<0, \quad p=1
$$

Therefore, the Equation (22) holds when the Equation (15) holds.

When $p=0$, the event of packet dropout does not occur. The random delay in the system is expressed through uncertain parameters. It follows from (20) that

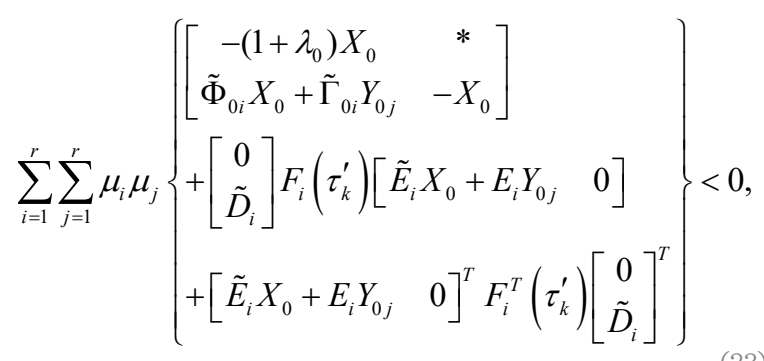

where $Y_{0 j}=K_{j} X_{0}$.

According to $F_{i}^{T}\left(\tau_{k}^{\prime}\right) F_{i}\left(\tau_{k}^{\prime}\right)<I$ and Lemma 3, the Equation (23) is equivalent to

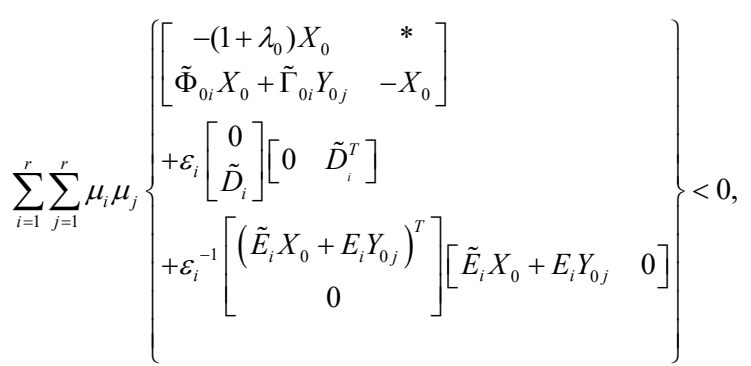

Appling Lemma 2, the Equation (24) is equivalent to

$$
\sum_{i=1}^{r} \sum_{j=1}^{r} \mu_{i} \mu_{j}\left[\begin{array}{ccc}
-\left(1+\lambda_{0}\right) X_{0} & * & * \\
\tilde{\Phi}_{0 i} X_{0}+\tilde{\Gamma}_{0 i} Y_{0 j} & \varepsilon_{i} \tilde{D}_{i} \tilde{D}_{i}^{T}-X_{0} & * \\
\tilde{E}_{i} X_{0}+E_{i} Y_{0 j} & 0 & -\varepsilon_{i} I
\end{array}\right]<0
$$

Therefore, the Equation (25) holds when Equation (14) holds.

If the MDADT of the switched system (9) satisfies (10), the exponential stability conditions of the fuzzy switched system (9) can be analyzed through Theorem 1 and the controller gains can obtained by solving LMIs (14)-(16) in Theorem 2.

Remark 2. The design parameters $\sigma_{i} \mu_{0}, \mu_{1}, \lambda_{0}, \lambda_{1}$ must firstly satisfy $\sigma_{i}>\left\|\int_{0}^{-\tau_{k}^{\prime}} e^{A_{i} s} d s\right\|_{2}, \mu_{0}>1,0<\mu_{1}<1$, $-1<\lambda_{0}<0,0<\lambda_{1}<1$. In addition, for the practical packet transmission time sequence, the design parameters should satisfy $\tau_{a 0} \geq \tau_{a 0}^{*}=-\frac{\ln \mu_{0}}{\ln \left(1+\lambda_{0}\right)} \quad$ and $\tau_{a 1} \leq \tau_{a 1}^{*}=-\frac{\ln \mu_{1}}{\ln \left(1+\lambda_{1}\right)}$. It is worth mentioning that the larger the packet loss rate of the system is, the more dif- 
ficult it is to obtain the proper controller gains which guarantees the exponential stability of the system state.

Remark 3. In the traditional average dwell time (ADT) method, all subsystems follow the same ADT. However, in the mode-dependent average dwell time (MDADT) method, each subsystem follows a separate $\mathrm{ADT}$, which can obtain less conservation results. In addition, the combination of the slow switching method and the fast method is a complement to the MDADT method, which brings more flexibility to the stability analysis.

Remark 4. It is worth mentioning that in real-word applications, practical systems always suffer from such issues as input saturation, input dead zones and unidirectional input constraints. In [16] and [22], the problem of input constraints and input dead zones is effectively investigated to reduce final positioning errors. In future work, we may consider employing robust control technique to solve the problem of input constraints.

\section{Numerical Simulation}

In this section, a rotary inverted pendulum (RIP) is used as an experimental test, and the performance of the proposed fuzzy controller is evaluated in the nonlinear NCS. To verify that T-S fuzzy model is more advantageous than linearization in coping with nonlinear systems, some experimental results of the fuzzy state feedback controller based on T-S fuzzy model and the linear state feedback controller based on linearization are compared.

The experiment system, as shown in figure 3 , consists of a Quanser RIP and a PC. The RIP is composed of a servo motor, a rotary and a pendulum link and its structure is shown in figure 4 . The controllers for the RIP are implemented in Matlab/Simulink. The mathematical model from the practical RIP is is developed using the Euler-Lagrange method. The nonlinear dynamics model of the RIP is described as

$$
\left\{\begin{array}{l}
\left(m_{p} L_{r}{ }^{2}+\frac{1}{4} m_{p} L_{p}^{2}-\frac{1}{4} m_{p} L_{p}^{2} \cos ^{2}(\alpha)+J_{r}\right) \ddot{\theta}-\frac{1}{2} m_{p} L_{p} L_{r} \cos (\alpha) \ddot{\alpha} \\
+\frac{1}{2} m_{p} L_{p}^{2} \sin (\alpha) \cos (\alpha) \dot{\theta} \dot{\alpha}+\frac{1}{2} m_{p} L_{p} L_{r} \sin (\alpha) \dot{\alpha}^{2}=\frac{k_{m}\left(V_{m}-k_{m} \dot{\theta}\right)}{R_{m}}-D_{r} \dot{\theta} \\
-\frac{1}{2} m_{p} L_{p} L_{r} \cos (\alpha) \ddot{\theta}+\left(J_{p}+\frac{1}{4} m_{p} L_{p}^{2}\right) \ddot{\alpha}-\frac{1}{4} m_{p} L_{p}^{2} \sin (\alpha) \cos (\alpha) \dot{\theta}^{2} \\
-\frac{1}{2} m_{p} L_{p} g \sin (\alpha)=-D_{p} \dot{\alpha}
\end{array}\right.
$$

Figure 3

The experiment system for the RIP

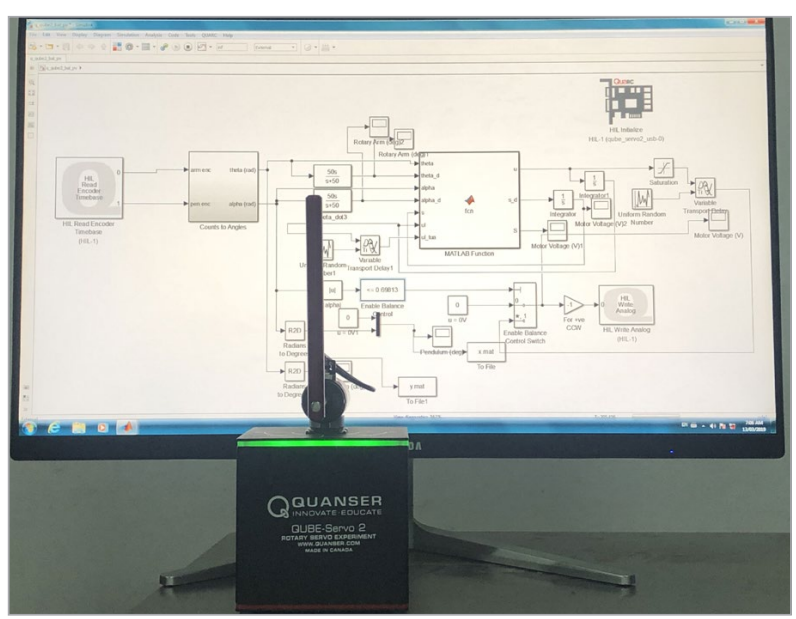

Figure 4

Free-body diagram of rotary pendulum

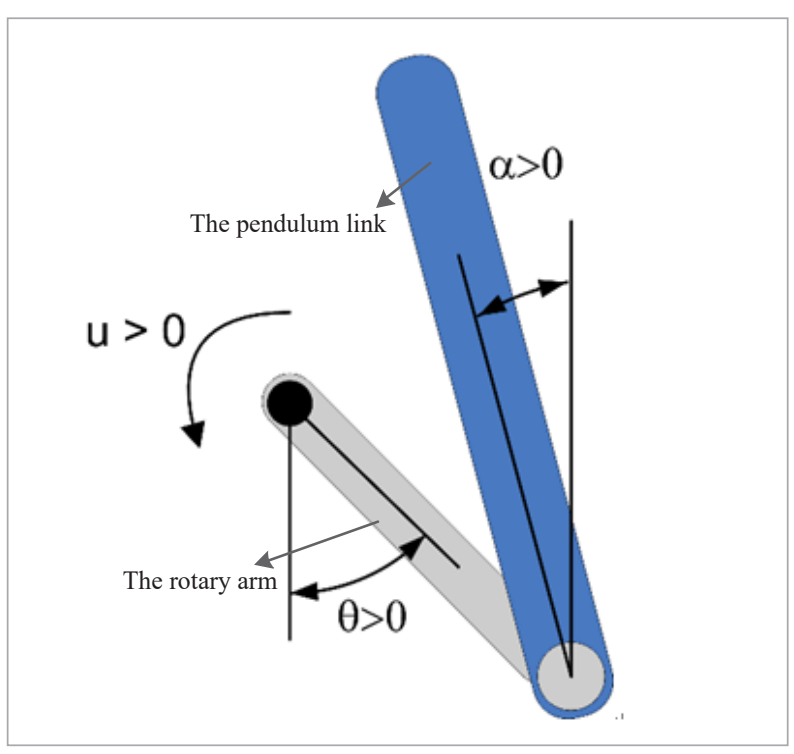

where $\theta$ is rotary arm angle, $\dot{\theta}$ is rotary arm angular velocity, $\alpha$ is pendulum link angle, $\dot{\alpha}$ is pendulum angular velocity, $V_{m}$ is the input voltage applied on the servo motor. Other system parameters are shown in Table 1. Supposed that the state vector $x=\left[\begin{array}{llll}\theta & \alpha & \dot{\theta} & \dot{\alpha}\end{array}\right]$ and the input $u=V_{m}$. For there is $\dot{\theta} \approx 0, \quad \dot{\alpha} \approx 0$ near the equilibrium point, the above nonlinear dynamics model can be simplified as

$$
E \dot{x}=A x+B u
$$




$$
\begin{aligned}
& E=\left[\begin{array}{cccc}
1 & 0 & 0 & 0 \\
0 & 1 & 0 & 0 \\
0 & 0 & \left(m_{p} L_{r}{ }_{r}+\frac{1}{4} m_{p} L_{p}{ }^{2}-\frac{1}{4} m_{p} L_{p}{ }^{2} \cos ^{2}(\alpha)+J_{r}\right) & -\frac{1}{2} m_{p} L_{p} L_{r} \cos (\alpha) \\
0 & 0 & -\frac{1}{2} m_{p} L_{p} L_{r} \cos (\alpha) & \left(J_{p}+\frac{1}{4} m_{p} L_{p}{ }^{2}\right)
\end{array}\right] \\
& A=\left[\begin{array}{cccc}
0 & 0 & 1 & 0 \\
0 & 0 & 0 & 1 \\
0 & 0 & -\left(\frac{k_{m}}{R_{m}}+D_{r}\right) & 0 \\
0 & \frac{1}{2} m_{p} L_{p} g \frac{\sin (\alpha)}{\alpha} & 0 & -D_{p}
\end{array}\right]
\end{aligned}
$$$$
B=\left[\begin{array}{llll}
0 & 0 & \frac{k_{m}}{R_{m}} & 0
\end{array}\right]^{T}
$$

To verify that T-S fuzzy model is more advantageous than linearization in coping with nonlinear systems, T-S fuzzy model and linearization are employed separately to represent the nonlinear dynamics model Equation (27)

\section{Table 1}

\begin{tabular}{|c|c|c|}
\hline Symbol & Description & Value \\
\hline$R_{m}$ & Terminal resistance & $8.4 \Omega$ \\
\hline$k_{m}$ & Motor back-emf constant & $0.042 \mathrm{~V} \cdot \mathrm{s} / \mathrm{rad}$ \\
\hline$m_{r}$ & Rotary arm mass & $0.095 \mathrm{~kg}$ \\
\hline$L_{r}$ & Rotary arm length & $0.085 m$ \\
\hline$J_{r}$ & $\begin{array}{l}\text { Moment of inertia } \\
\text { about the arm }\end{array}$ & $5.720 \times 10^{-5} \mathrm{~kg} \cdot \mathrm{m}^{2}$ \\
\hline$D_{r}$ & $\begin{array}{c}\text { Friction coefficient } \\
\text { of the arm }\end{array}$ & $0.0015 \mathrm{~N} \cdot \mathrm{m} \cdot \mathrm{s} / \mathrm{rad}$ \\
\hline$m_{p}$ & Pendulum link mass & $0.024 \mathrm{~kg}$ \\
\hline$L_{P}$ & Pendulum link length & $0.129 m$ \\
\hline$J_{p}$ & $\begin{array}{l}\text { Moment of inertia about the } \\
\text { Pendulum link }\end{array}$ & $3.328 \times 10^{-5} \mathrm{~kg} \cdot \mathrm{m}^{2}$ \\
\hline$D_{p}$ & $\begin{array}{l}\text { Friction coefficient of the } \\
\text { pendulum }\end{array}$ & $0.0005 \mathrm{~N} \cdot \mathrm{m} \cdot \mathrm{s} / \mathrm{rad}$ \\
\hline$g$ & Gravity & $9.81 m / s^{2}$ \\
\hline
\end{tabular}

Physical parameters of the RIP

\section{T-S fuzzy model}

There are $\frac{\sin (\alpha)}{\alpha} \approx 1$ and $\cos (\alpha) \approx 1$ when $\alpha$ approaches 0. And there are $\frac{\sin (\alpha)}{\alpha} \approx \pm \frac{2 \sqrt{2}}{\pi}$ and $\cos (\alpha) \approx \pm \frac{\sqrt{2}}{2}$ when $\alpha$ approaches $\pm 45^{\circ}$. In order to reduce the number of model rules, the local approximation approach in fuzzy partition spaces is used to obtain T-S fuzzy model of nonlinear model Equation (27). Thus, according to Equation (2), its fuzzy rules are

Rule 1: IF $\alpha$ is about 0, THEN $\dot{x}=A_{1} x+B_{1} u$.

Rule 2: IF $\alpha$ is about $\pm \pi / 4(|\alpha|<\pi / 4)$, THEN $\dot{x}=A_{2} x+B_{2} u$.

Substituting the values in Table 1 into the above coefficient matrices, there are

$$
\begin{gathered}
A_{1}=\left[\begin{array}{cccc}
0 & 0 & 1 & 0 \\
0 & 0 & 0 & 1 \\
0 & 149.2751 & -14.9287 & -4.0149 \\
0 & 261.6091 & -14.7551 & -8.6163
\end{array}\right], B_{1}=\left[\begin{array}{c}
0 \\
0 \\
49.7275 \\
49.1493
\end{array}\right] \\
A_{2}=\left[\begin{array}{cccc}
0 & 0 & 1 & 0 \\
0 & 0 & 0 & 1 \\
0 & 44.3406 & -7.9352 & -1.6216 \\
0 & 133.6878 & -5.5458 & -4.8891
\end{array}\right], B_{2}=\left[\begin{array}{c}
0 \\
0 \\
23.2023 \\
18.2157
\end{array}\right]
\end{gathered}
$$

The system defines two Triangle-shape membership functions, shown in Figure 5. The membership functions of Rule 1 and Rule 2 are chosen as

\section{Figure 5}

Triangle-shape membership functions

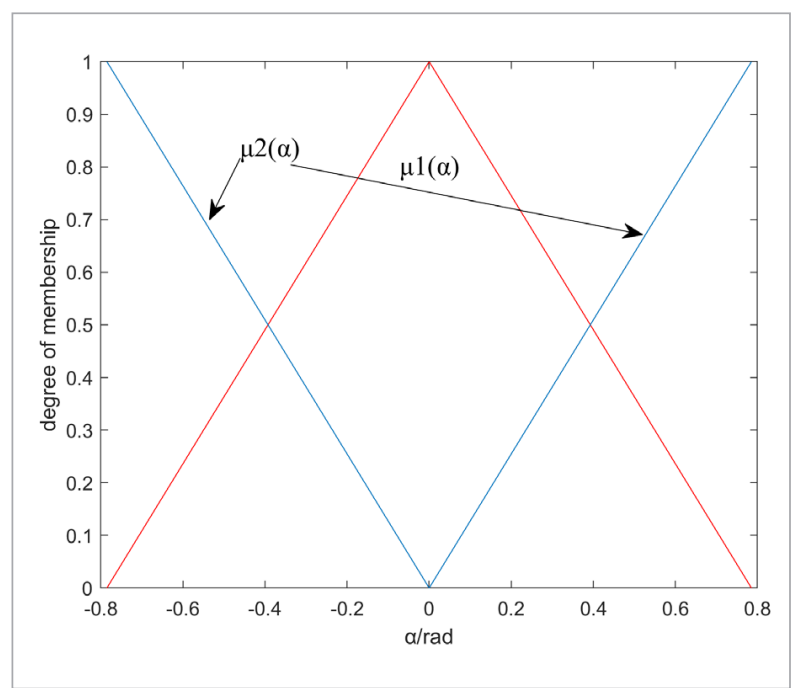




$$
\mu_{1}(\alpha)=\left\{\begin{array}{l}
-\frac{4}{\pi} a+1,0<a \leq \frac{\pi}{4} \\
\frac{4}{\pi} a+1,-\frac{\pi}{4} \leq a \leq 0
\end{array} \text { and } \mu_{2}(\alpha)=1-\mu_{1}(\alpha) .\right.
$$

Therefore, according to Equation (2), the nonlinear model Equation (27) can be written as a fuzzy model

$$
\dot{x}(t)=\sum_{i=1}^{2} \mu_{i}(\alpha)\left[A_{i} x(t)+B_{i} u(t)\right] .
$$

\section{Model linearization}

When $\alpha$ approaches 0, the nonlinear model Equation (28) can be expressed as a linear model

$$
\dot{x}=A x+B u,
$$

where

$$
A=\left[\begin{array}{cccc}
0 & 0 & 1 & 0 \\
0 & 0 & 0 & 1 \\
0 & 149.2751 & -14.9287 & -4.0149 \\
0 & 261.6091 & -14.7551 & -8.6163
\end{array}\right], B=\left[\begin{array}{c}
0 \\
0 \\
49.7275 \\
49.1493
\end{array}\right]
$$

According to the above two models, the fuzzy state feedback controller and the linear state feedback controller are applied to control the RIP system respectively. The control effects of the two control methods are analyzed and compared.

The sampling period of the sensor is chosen as $T=0.02 s$. For other parameters, we have $\sigma_{1}=\sigma_{2}=0.080, \mu_{0}=1.850, \mu_{1}=0.550, \lambda_{0}=-0.116$, $\lambda_{0}=0.700$. According to (10), we can derive that $\tau_{a 0}^{*}=4.9894, \tau_{a 1}^{*}=1.1267$. For the above T-S fuzzy model Equation (27), solving LMIs (14), (15) and (16), by theorem 2 , we can get controller gain matrices

$$
K_{1}=\left[\begin{array}{llllll}
0.1401 & -12.3400 & 0.4601 & -0.7656 & 0.0358
\end{array}\right] \text {, }
$$

$K_{2}=\left[\begin{array}{llllll}0.4084 & -18.9397 & 0.6594 & -1.3042 & -0.0819\end{array}\right]$. For the above linear model Equation (28), similarly, we can get controller gain matrix

$$
K=\left[\begin{array}{lllll}
0.2067 & -12.8365 & 0.5122 & -0.8100 & 0.0203
\end{array}\right] \text {. }
$$

Suppose that random delay satisfies $\tau_{k}=\tau_{k}^{s c}+\tau_{k}^{c a} \in(0,0.02 s)$ and the time sequence diagram of packet transmission is shown in figure 6 . Then the corresponding MDADT for packet-dropout and packet-send cases respectively are $\tau_{a 0}=5.0487>\tau_{a 0}^{*}$ and $\tau_{a 1}=1.0750<\tau_{a 1}^{*}$, which satisfies the condi- tions of MDADT in the Equation (10). According to Theorem 1, the exponential decay rate $\gamma$ satisfies $\gamma=\max \left\{\left(\mu_{0}^{\frac{1}{\tau_{a 0}}} \overline{\lambda_{0}}\right),\left(\mu_{1}^{\frac{1}{\tau_{11}}} \overline{\lambda_{1}}\right)\right\}<1$ and the constant $a$ satisfies $a=\frac{L \beta_{2}}{\beta_{1}}>0$. Therefore, the controlled system is exponentially stable. Figures 7 and 8 shows the experiment results of the fuzzy controller and linear controller, where $\alpha_{0}$ is the initial state of the pendulum link.

It can be seen from the control effects obtained by the above two different controllers. The fuzzy controller can balance the pendulum link for initial conditions $\alpha_{0} \in\left[\begin{array}{ll}-30^{\circ} & 30^{\circ}\end{array}\right]$ and the state of the system becomes unstable when $\left|\alpha_{0}\right|>30$. In contrast, the linear controller can only balance the pendulum for initial conditions $\alpha_{0} \in\left[\begin{array}{ll}-15^{\circ} & 15^{\circ}\end{array}\right]$ and the state of the system becomes unstable when $\left|\alpha_{0}\right|>15$. Therefore, the experiment results illustrate that the effectiveness of the proposed method for the NNCS with random delay and packet losses, and verify that the fuzzy controller based on T-S fuzzy model can balance the system in a larger stability range than the linear controller based on model linearization. However, there are some such issues as external disturbances in the system, input constrains and model errors between the established model and the practical nonlinear model that may lead to positioning errors during the entire control process in the experiments.

\section{Figure 6}

Packet transmission time sequence diagram with loss rate of $17.20 \%$ under sampling period $T=0.02 \mathrm{~s}$

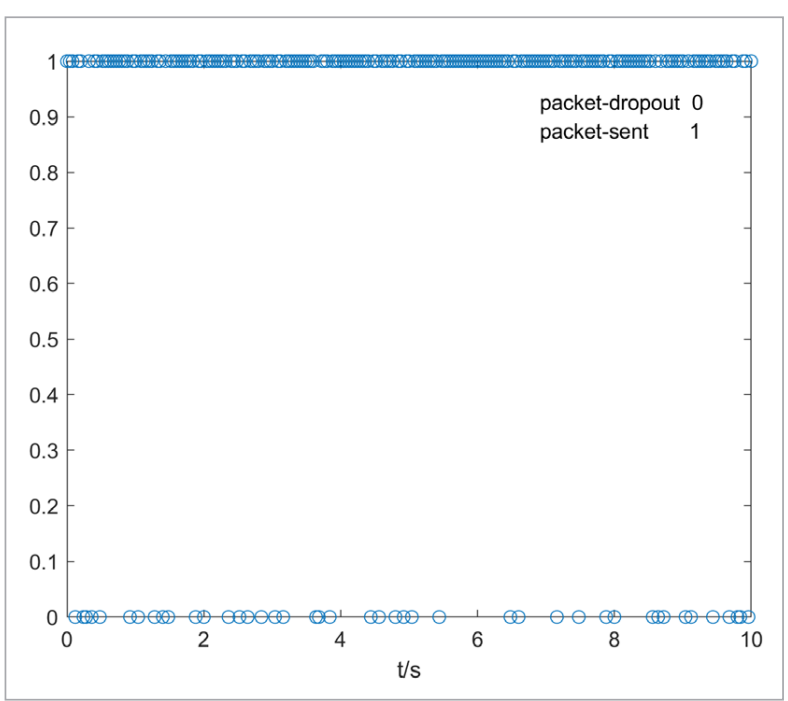


Figure 7

The experiment result of the fuzzy controller

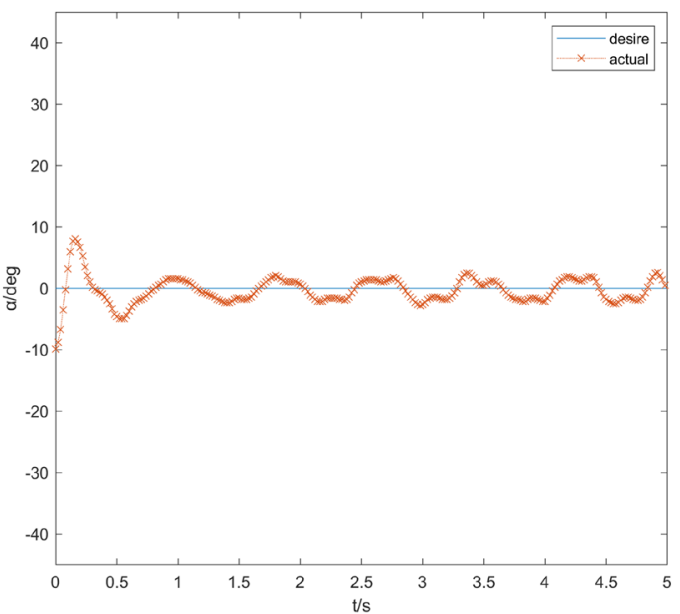

(a) $\alpha_{0}=-10^{\circ}$

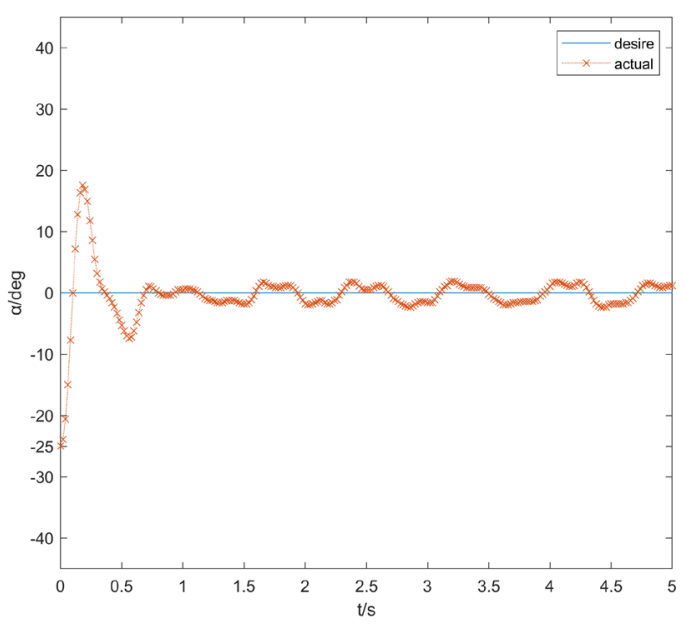

(c) $\alpha_{0}=-25^{\circ}$

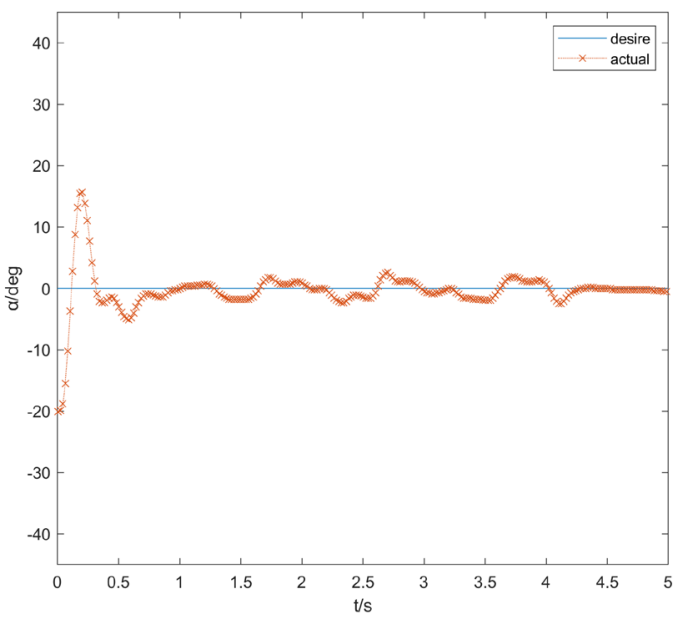

(b) $\alpha_{0}=-20^{\circ}$

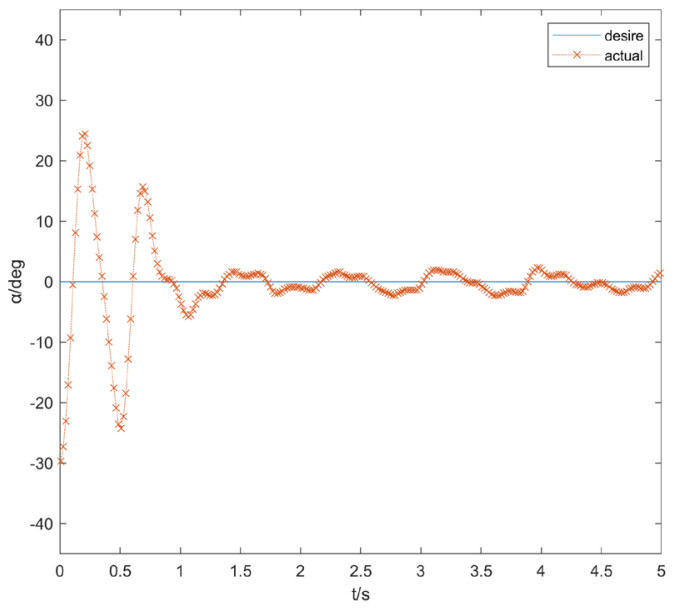

(d) $\alpha_{0}=-30^{\circ}$

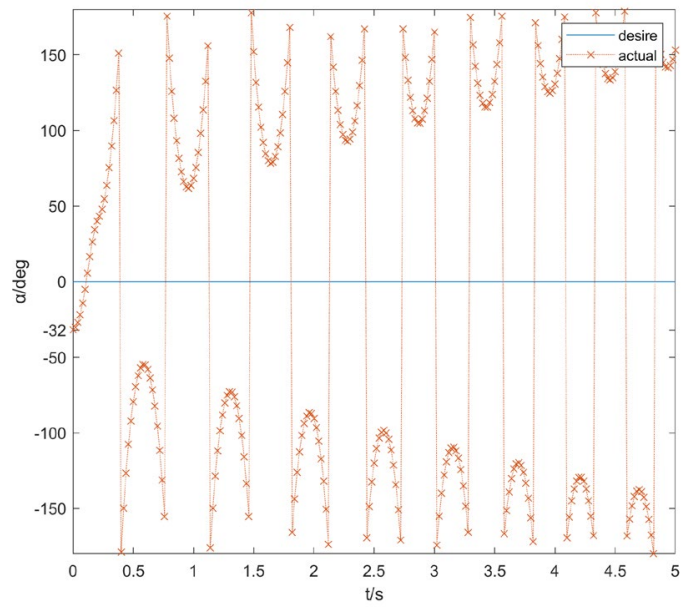

(e) $\alpha_{0}=-32^{\circ}$ 


\section{Figure 8}

The experiment result of the linear controller

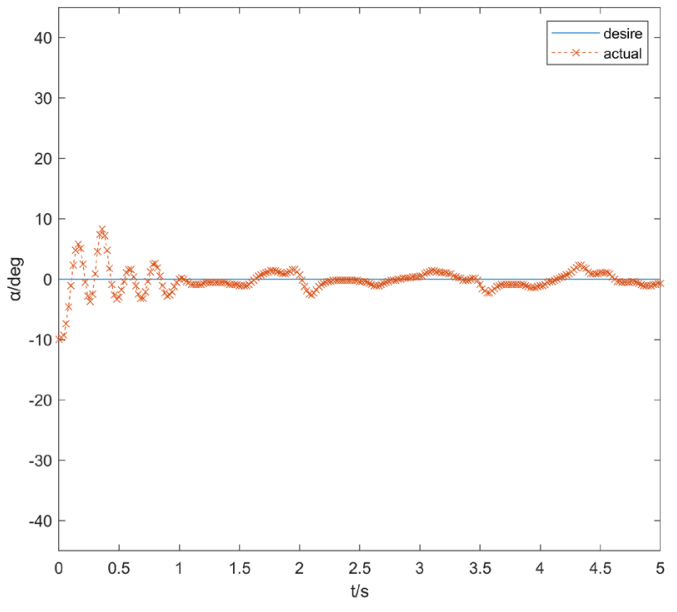

(a) $\alpha_{0}=-10^{\circ}$

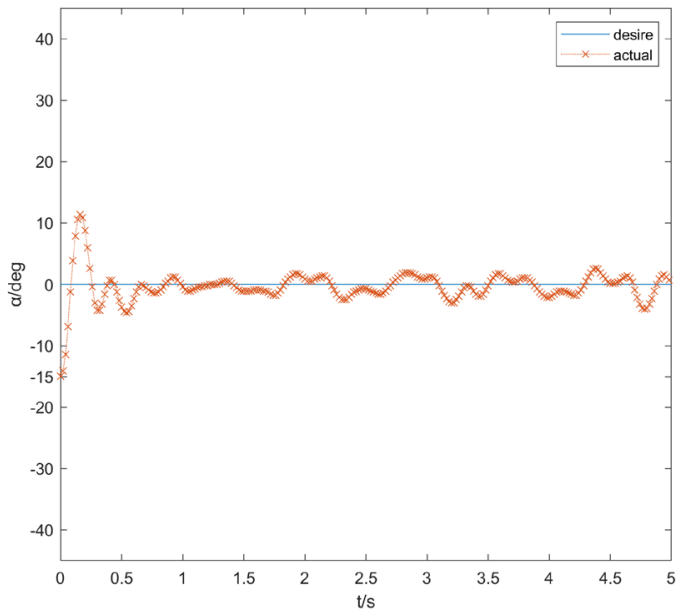

(b) $\alpha_{0}=-15^{\circ}$

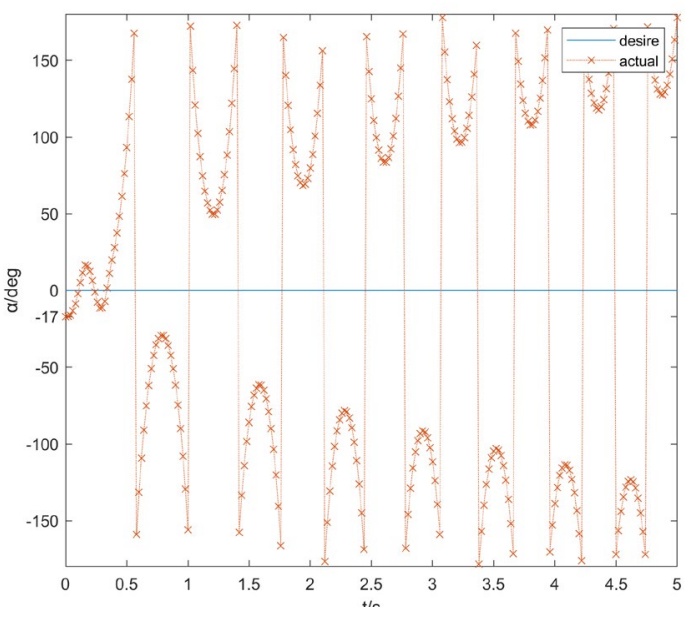

(c) $\alpha_{0}=-17^{\circ}$

\section{Conclusion}

In this paper, the NNCS with bilateral random delays and packet losses are modeled as a discrete-time fuzzy switched system with parameter uncertainty. The combination of slow switching and fast switching MDADT methods is used to give the state stability condition of the switched system. The fuzzy controller gains are obtained by solving corresponding LMIs. Experiment results illustrate the feasibility of the proposed method and prove T-S fuzzy model is more advantageous than linearization in coping with nonlinear systems. Future works could focus on some problems such as various external disturbances in the system; input constrains and model errors between the T-S fuzzy model and practical nonlinear model.

\section{Acknowledgement}

The work sponsored by the Hubei Province Key Laboratory of Systems Science in Metallurgical Process (Z201702). 


\section{References}

1. Guan, R., Jiang, X., Li, Y. Controller Design Method for Discretization of T-S Fuzzy Control System. Journal of Hangzhou Dianzi University, 2013, 33(3), 49-52. http:// dx.doi.org/10.3969/j.issn.1001-9146.2013.03-013

2. Han, F., Feng, G., Wang, Y., Qiu, J., Zhang, C, Z. A Novel Dropout Compensation Scheme for Control of Networked T-S Fuzzy Dynamic Systems. Fuzzy Sets and Systems, 2014, 235, 44-61. https://doi.org/10.1016/j. fss.2013.05.011

3. He, J, Y., Zheng, Y. H $\infty$ Mean-Square Exponentially Stable Control for a Class of Networked Control Systems with Time Delay and Packet Dropout. Journal of Hebei Normal University, 2017, 7(2), 116-123. https://doi. org/10.1016/j.fss.2013.05.011

4. He, Y., Zhang, Y., Wu M. Improved Exponential Stability for Stochastic Markovian Jump Systems with Nonlinearity and Time-Varying Delay. International Journal of Robust \& Nonlinear Control, 2010, 20(1), 16-26. https://doi.org/10.1002/rnc.1412

5. Hu, S., Zhang, Y., Yin, X., Du Z. T-S Fuzzy-Model-Based Robust Stabilization for A Class of Nonlinear Discrete-Time Networked Control Systems. Nonlinear Analysis: Hybrid Systems, 2013, 8, 69-82. https://doi. org/10.1016/j.nahs.2012.11.001

6. Li, H., Wu, C., Shi, P., Gao Y. Control of Nonlinear Networked Systems with Packet Dropouts: Interval Type2 Fuzzy Model-Based Approach. IEEE Transactions on Cybernetics, 2015, 45(11), 2378-2389. https://doi. org/10.1109/TCYB.2014.2371814

7. Li, T, X., Zhang, W, A., Li, Y. Improved Switched System Approach to Networked Control Systems with Time-Varying Delays. IEEE Transactions on Control Systems Technology, 2019, 27(6), 2711-2717. https:// doi.org/10.1109/TCST.2018.2859901

8. Li, Y., Zhang, H. Asynchronous Hœ Control of Switched Uncertain Discrete-Time Fuzzy Systems via Basis-Dependent Multiple Lyapunov Functions Approach. Circuits Systems \& Signal Processing, 2018, 37(4), 135162. https://doi.org/10.1007/s00034-017-0550-5

9. Lin, Q., Chen, S., Chai, Q. Dissipative Fuzzy Control of Nonlinear Networked Control Systems with Multiple Packet Dropout. Australian and New Zealand Control Conference (ANZCC). IEEE, 2017, 41-46. https://doi. org/10.1109/ANZCC.2017.8298441

10. Liu, B., Liu, Y, C., Li, W., Ma, Y, J., Jang, Z. Modeling and Control of Networked Control Systems with Random Network-Induced Delay and Packet-Dropout. 12th
IEEE Conference on Industrial Electronics and Applications (ICIEA). IEEE, 2017, 786-791. https://doi. org/10.1109/ICIEA.2017.8282946

11. Liu, L., Yin, Y., Wang, J., Wu, Q. Stability Analysis of Discrete-Time Switched Nonlinear Systems via T-S Fuzzy Model Approach. Neurocomputing, 2016, 173(P3), 1967-1971. https://doi.org/10.1016/j.neucom.2015.08.007

12. Liu, Y, C., Liu, B. Research on Networked Switching System Control with Delay and Packet Loss. Scientific Journal of Control Engineering, 2018, 25(8), 1482-1489. http://dx.doi.org/10.14107/j.cnki.kzgc.160777r

13. Luo, J., Zhao, J. Robust $\mathrm{H} \infty$ Control for Networked Switched Fuzzy Systems with Network-Induced Delays and Packet Dropout. Circuits, Systems, and Signal Processing, 2015, 34(2), 663-679. https://doi.org/10.1007/ s00034-014-9860-z

14. Marouf, S., Mahboobi, E, R., Akbari, A., Barforooshan, M. T-S Fuzzy Controller Design for Stabilization of Nonlinear Networked Control Systems. Engineering Applications of Artificial Intelligence, 2016, 50, 135141. https://doi.org/10.1016/j.engappai.2016.01.031

15. Qiu, J., Gao, H., Ding, S, X. Recent Advances on FuzzyModel-Based Nonlinear Networked Control Systems: A Survey. IEEE Transactions on Industrial Electronics, 2015, 63(2), 1207-1217. https://doi.org/10.1109/ TIE.2015.2504351

16. Sun, N., Liang, D., Wu, Y., Chen, Y., Qin, Y., Fang, Y. Adaptive Control for Pneumatic Artificial Muscle Systems with Parametric Uncertainties and Unidirectional Input Constraints. IEEE Transactions on Industrial Informatics, 2020, 16(2), 967-979. https://doi. org/10.1109/TII.2019.2923715

17. Tanaka, K., Wang, H, O. Fuzzy Control Systems Design and Analysis: A Linear Matrix Inequality Approach. John Wiley \& Sons, Inc. 2002, 23-31. https://doi. org/10.1016/S0005-1098(03)00188-2

18. Wang, J., Yang, H. Exponential Stability of A Class of Networked Control Systems with Time Delays and Packet Dropouts[J]. Applied Mathematics and Computation, 2012, 218(17), 8887-8894. https://doi. org/10.1016/j.amc.2012.02.0477

19. Wang, M., Qiu, J., Chadli, M., Wang, M. A Switched System Approach to Exponential Stabilization of Sampled-Data T-S Fuzzy Systems with Packet Dropouts. IEEE Transactions on Cybernetics, 2016, 46(12), 31453156. https://doi.org/10.1109/TCYB.2015.2498522 
20. Xu, D., Li, Z., Chen, H. Event-Triggered Hœ Control for Discrete-Time Networked T-S Fuzzy Systems. Control Conference. IEEE, 2017, 7719-7724. https://doi. org/10.23919/ChiCC.2017.8028577

21. Xue, M., Tang, Y., Wu,Li. Switching Stabilization for Type2 Fuzzy Systems with Network-Induced Packet Losses. IEEE Transactions on Cybernetics, 2019, 49(7), 25912604. https://doi.org/10.1109/TCYB.2018.2829487

22. Yang, T., Sun, N., Chen, H., Fang, Y, C., Neural Network-Based Adaptive Antiswing Control of an Underactuated Ship-Mounted Crane with Roll Motions and Input Dead Zones. IEEE Transactions on Neural Networks and Learning Systems, 2020, 31(3), 901-914. https://doi.org/10.1109/TNNLS.2019.2910580

23. Yong, Z., Fang, H. Stabilization of Nonlinear Networked Systems with Sensor Random Packet Dropout and Time-Varying Delay. Applied Mathematical Modelling, 2011, 35(5), 2253-2264. https://doi.org/10.1016/j. apm.2010.11.038

24. Zhang, H., Fang, H., Ren, X. Stability Analysis of Networked Control System Based on Quasi T-S Fuzzy Mod- el. International Journal of Modelling Identification and Control, 2012, 16(1), 41-49. https://doi.org/10.1504/ IJMIC.2012.046694

25. Zhang, H., Xie, D., Zhang, H., Wang, G. Stability Analysis for Discrete-Time Switched Systems with Unstable Subsystems by a Mode-Dependent Average Dwell Time Approach. ISA Transactions, 2014, 53(4), 1081-1086. https://doi.org/10.1016/j.isatra.2014.05.020

26. Zhang, W, A., Yu, L. Modelling and Control of Networked Control Systems with Both Network-Induced Delay and Packet-Dropout. Automatica, 2008, 44(12), 32063210. https://doi.org/10.1016/j.automatica.2008.09.001

27. Zhang, X., Han, Q., Yu, X. Survey on Recent Advances in Networked Control Systems. IEEE Transactions on Industrial Informatics, 2017, 12(5), 1740-1752. https://doi. org/10.1109/TII.2015.2506545

28. Zhi, L., He, Y., Zhang, C, K. Improved Stabilization Conditions for T-S Fuzzy Systems with Interval Time-Varying Delay. Proceedings of the 36th Chinese Control Conference (CCC2017). 2017, 294-389. https:// doi.org/10.23919/ChiCC.2017.8027372 